\title{
The Influence of Flightless I on Toll-Like-Receptor-Mediated Inflammation in a Murine Model of Diabetic Wound Healing
}

\author{
Nadira Ruzehaji, ${ }^{1,2}$ Stuart J. Mills, ${ }^{1}$ Elizabeth Melville, ${ }^{1}$ Ruth Arkell, ${ }^{3}$ \\ Robert Fitridge, ${ }^{2}$ and Allison J. Cowin ${ }^{1,2}$ \\ ${ }^{1}$ Wound Healing Laboratory, Women's \& Children's Health Research Institute, 72 King William Road, \\ North Adelaide, Adelaide, SA 5006, Australia \\ ${ }^{2}$ Faculty of Health Sciences, The University of Adelaide, Adelaide, SA 5005, Australia \\ ${ }^{3}$ Early Mammalian Development Laboratory, Research School of Biological Sciences, Australian National University, \\ Canberra, ACT 2601, Australia
}

Correspondence should be addressed to Allison J. Cowin; allison.cowin@adelaide.edu.au

Received 25 October 2012; Revised 7 January 2013; Accepted 7 January 2013

Academic Editor: Jorge Berlanga Acosta

Copyright (C) 2013 Nadira Ruzehaji et al. This is an open access article distributed under the Creative Commons Attribution License, which permits unrestricted use, distribution, and reproduction in any medium, provided the original work is properly cited.

Impaired wound healing and ulceration represent a serious complication of both type 1 and type 2 diabetes. Cytoskeletal protein Flightless I (Flii) is an important inhibitor of wound repair, and reduced Flii gene expression in fibroblasts increased migration, proliferation, and adhesion. As such it has the ability to influence all phases of wound healing including inflammation, remodelling and angiogenesis. Flii has the potential to modulate inflammation through its interaction with MyD88 which it an adaptor protein for TLR4. To assess the effect of Flii on the inflammatory response of diabetic wounds, we used a murine model of streptozocininduced diabetes and Flii genetic mice. Increased levels of Flii were detected in Flii transgenic murine wounds resulting in impaired healing which was exacerbated when diabetes was induced. When Flii levels were reduced in diabetic wounds of Flii-deficient mice, healing was improved and decreased levels of TLR4 were observed. In contrast, increasing the level of Flii in diabetic mouse wounds led to increased TLR 4 and NF- $\kappa$ B production. Treatment of murine diabetic wounds with neutralising antibodies to Flii led to an improvement in healing with decreased expression of TLR4. Decreasing the level of Flii in diabetic wounds may therefore reduce the inflammatory response and improve healing.

\section{Introduction}

Up to $25 \%$ of people with diabetes can expect to develop a foot ulcer at some point in their lives [1]. Due to poor outcomes of existing therapies, lower extremity amputation is a common complication, affecting $15 \%$ of diabetics with foot ulcers, with one major amputation occurring every 30 seconds worldwide and over 2500 limbs lost a day [2]. The effectiveness of current treatments for diabetic foot ulcers is limited, and many patients with chronic, unhealed wounds need continual care. Understanding the processes involved in impaired wound healing will help to develop new therapeutic targets and tools for improving wound repair.

One area of research which has been shown to be integral to the wound repair process is that of the actin cytoskeleton which is a filamentous network found in all cells and facilitates processes such as cellular adhesion, migration and contraction [3, 4]. One member of the actin cytoskeleton is Flightless I (Flii) which is a member of the gelsolin family of actin remodelling proteins [5]. Flii colocalizes with actin and microtubule-based structures, is required for normal actin distribution, and possesses $\mathrm{Ca}^{2+}$-independent $\mathrm{G}$-actin binding activity as well as F-actin binding and severing activities $[3,6-8]$. In addition to its role as a regulator of the cytoskeleton, the LRR domain allows Flii to bind a number of other proteins unrelated to actin including LRR Flii interacting proteins 1 and 2 (LLRFIP1 and LRRFIP2) and the double-stranded RNA binding protein TRIP $[4,9]$. Flii is involved in numerous cellular activities including regulating transcription via coactivation of nuclear hormone 
receptors $[10,11]$ and regulation of beta-catenin-dependent transcription [12], important signalling pathways including the TLR pathway $[13,14]$, cellular polarity, asymmetric cell division [15], proliferation via interactions with calmodulindependent protein kinase type II [16], and inflammation and cytokine production via caspase activation and IL- $1 \beta$ maturation [17]. Flii-deficient mice have improved reepithelialisation after wounding while Flii overexpressing mice have impaired healing with larger wounds with reduced contraction, cellular proliferation and delayed reepithelialisation [18].

Increased inflammation is an important contributing factor in the failure to heal of diabetic foot ulcers [19]. Inflammation is an integral part of the wound healing process and is regulated by toll-like receptors (TLRs). TLRs are key innate immune receptors that alert the immune system to tissue damage and mediate the inflammatory response. The human TLR family consists of 10 members structurally characterised by the presence of a leucine-rich repeat (LRR) domain in their extracellular domain and toll/interleukin(IL-) 1 receptor (TIR) domain, in their intracellular domain. Through their intracellular TIR domain TLRs activate or deactivate signalling pathways that generate cytokine and chemokine production and thereby regulate inflammatory responses. TLR signalling is tightly regulated to control the intensity and duration of inflammation [12]. TLRs are expressed on a wide variety of cells including macrophages and neutrophils, and they respond to an array of viral, bacterial, and fungal ligands as well as cellular debris [13]. The receptors convey their specificity through the utilization of different adaptor proteins such as myeloid differentiation factor 88 (MyD88), TIR-associated protein (TIRAP), TIR domain containing adaptor protein-inducing IFN- $\beta$ (TRIF), and TRIF-related adaptor molecule (TRAM) [13-15]. Upon activation, the adaptor proteins promote signalling to result in the expression of proinflammatory cytokines, growth factors, and interferons. MyD88 has been shown to play an important role during wound healing as MyD88 knockout mice have impaired wound healing [16] with wounds showing reduced contraction, decreased and delayed granulation tissue formation, and reduced blood vessel density [16].

The LRR region of Flii shares 29\% sequence identity and 42\% similarity to TLR4 [20]. Through its interaction with MyD88, it has been suggested that Flii can modulate inflammation by suppressing TLR4-MyD88-mediated activation of $\mathrm{NF}-\kappa \mathrm{B}$ [21]. Conversely, a reduction in the Flii level may enhance activation of NF- $\kappa \mathrm{B}$ and increase cytokine secretion [20]. Several studies investigating the effect of Flii on TLR signalling in murine macrophages suggest that Flii can sequester activator proteins such as LPS and adaptor proteins such as MyD88 resulting in reduced cytokine expression $[10,11,16,17]$. In this study we used mice with low $\left(\mathrm{Flii}^{+/-}\right)$, normal (WT), and high (Flii ${ }^{\mathrm{Tg} / \mathrm{Tg}}$ ) Flii gene expression levels to investigate the function of Flii in a murine wound healing model of streptozocin-induced type 1 diabetes. We also investigated whether modulation of Flii by exogenous application of Flii neutralising antibodies improved diabetic wound healing via effects on TLR-mediated inflammation.

\section{Materials and Methods}

2.1. Antibodies. Mouse monoclonal anti-Flii antibodies raised to the LRR domain of the human Flii protein and the rabbit anti-human MyD88 antibody were obtained from Santa Cruz Biotechnology (VIC, Australia). Rabbit anti-human TLR4 and TLR9 antibodies were obtained from Imgenex (SA, Australia), mouse anti-human CD14 antibody and mouse anti-human CD16 antibody from BD Biosciences (NSW, Australia), and rabbit anti-human NF- $\kappa$ B antibody from Abcam (NSW, Australia). All antibodies were used at a 1:100 dilution. The appropriate secondary antibodies were used depending on the fluorescence required-goat anti-mouse Alexa Fluor 488 and goat anti-mouse Alexa Fluor 594 were obtained from Life Technologies (VIC, Australia) and also used at a 1:100 dilution. Flii is a highly conserved protein with $95 \%$ homology between mice and humans [19]. The Flii neutralising antibodies (FnAbs) used in in vivo diabetic mouse trials were mouse monoclonal anti-Flii antibodies raised against the N-terminus of the LRR domain of the human Flii protein [22] and obtained from Monoclonal Antibody SA Technologies (SA, Australia). Mouse IgG antibody used as a control in vivo diabetic mouse was obtained from Sigma (MO, USA)

2.2. Animal Studies. All experiments were approved by the Women's and Children's Health Network Animal Ethics Committee following the Australian Code of Practice for the Care and the Use of Animals for Scientific Purposes. Studies were performed using mice with a BALB/c background. Three strains of mice were used in this study with low $\left(\mathrm{Flii}^{+/-}\right.$), normal (WT), and high (Flii ${ }^{\mathrm{Tg} / \mathrm{Tg}}$ ) Flii gene expression levels. Mice lacking one copy of the Flii gene, the double knockout being embryonically lethal [23], were made as described previously [23] and will be written as Flii $^{+/-}$. Transgenic mice carrying two extra copies of human Flii gene incorporated into mouse genome will be written as Flii ${ }^{\mathrm{Tg} / \mathrm{Tg}}$.

2.3. Murine Model of Diabetic Wound Healing. Six female $\mathrm{Flii}^{+/-}$, WT, and Flii ${ }^{\mathrm{Tg} / \mathrm{Tg}}$ mice of 12-16 weeks of age and weighing 20-35 g were used for the induction of diabetes. Streptozocin (STZ) was used to induce type 1 diabetes (Sigma-Aldrich, MO, USA). STZ is toxic to the pancreatic beta-islet cells, rendering the mouse unable to produce adequate amount of insulin. Mice were given one intraperitoneal (IP) injection of STZ for 5 consecutive days (STZ: $50 \mathrm{mg} / \mathrm{kg}$ ) in citrate buffer of $\mathrm{pH}$ 6.5. This dose was chosen based on previously reported studies [24]. Age-matched nondiabetic control animals were treated with an equivalent dose of vehicle (citrate buffer alone). Diabetic symptoms were observed closely, and nonfasting blood glucose levels (BGLs) were tested weekly by tail vein sampling. To maintain body weight and prevent ketoacidosis, animals with confirmed diabetes were maintained with subcutaneous injection of insulin (1 IU, Mixtard 30/70, Novo-Nordisk, NSW, Australia). Mice were tested for sufficient levels of hyperglycaemia at 6 weeks after 
the last STZ injection, and only those with blood glucose levels greater than $15 \mathrm{mmol} / \mathrm{L}$ were wounded. Diabetic animals were wounded using a $6 \mathrm{~mm}$ biopsy punch (Stiefel Laboratories, NSW, Australia). Anaesthesia was induced by inhalation of isoflurane $(5 \%$ induction at $2 \mathrm{~L} / \mathrm{min}$ and $2 \%$ maintenance at $500 \mathrm{~mL} / \mathrm{min})$. To expose the skin, hair was removed by shaving and then application of hair removal cream (Veet, Reckitt Benckiser, NSW, Australia). Two $6 \mathrm{~mm}$ full thickness wounds, one on each side of the midline, were created on the dorsum of the mouse. Temgesic (buprenorphine $0.05 \mathrm{mg} / \mathrm{kg}$ ) was administered post-operatively to provide analgesia for up to 8 hours. The mice were euthanized at 7 days following wounding. Digital photographs of the wounds were taken at 0 and 7 days after wounding. A ruler was aligned next to the wound to allow direct wound area measurements to be made. Wounds were fixed in formalin and processed for histology and immunohistochemistry.

An additional cohort of female WT diabetic mice were injected intradermally around the wound margins with $200 \mu \mathrm{L}$ of FnAb $(50 \mu \mathrm{g} / \mathrm{mL} ; n=10)$ or mouse IgG control $(50 \mu \mathrm{g} / \mathrm{mL} ; n=10)$ immediately after surgery and at 1 and 2 days after wounding. Digital photographs of wounds were taken at days 0 and 7 after wounding. All animals were euthanized at day 7 post-wounding with the wounds harvested, fixed in formalin, and processed for histological analysis.

\subsection{Histology, Immunohistochemistry, and Image Analysis.} Histological sections $(4 \mu \mathrm{m})$ of mouse wounds were cut and stained with haematoxylin and eosin or subjected to immunohistochemistry following antigen retrieval as described previously [25]. Briefly, following antigen retrieval, sections were blocked in $3 \%$ normal goat serum, primary antibodies against Flii (1:100), TLR9 (1:100), TLR4 $(1: 100)$, MyD88 $(1: 100)$ and NF- $\kappa \mathrm{B}(1: 100)$ were applied and incubated overnight at $4^{\circ} \mathrm{C}$. Appropriate Alexa Fluor 488conjugated secondary antibodies $(1: 100)$ were used and incubated for 1 hour. Fluorescence intensity was determined using AnalySIS software package (Soft Imaging System GmbH, Munster, Germany), and optical fluorescence in the epidermis and dermis of the wounds was analysed as previously described [25]. Negative controls included replacing primary antibodies with normal mouse or normal rabbit IgG. Primary or secondary antibodies were omitted to verify the staining and detect nonspecific binding. All control sections had negligible immunofluorescence.

2.5. Histological Image Analysis. Histological image analysis was performed using the Digital Microscope Camera ProgRes C5 (JENOPTIK Laser, Jena, Germany). Wound size was determined by manually drawing below the clot or the portions of the wound that were not covered by epidermis. Dermal wound gape was determined by measuring between the dermal wound margins. Fluorescent images were taken using an Olympus IX81 (Olympus Australia, Melbourne, VIC, Australia) at a magnification of $\times 20$. The fluorescent intensity of the staining was calculated using Image Pro-Plus software (Media Cybernetics, MD, USA).

2.6. Statistical Analysis. Statistical significance was calculated using a paired Students $t$-test or analysis of variance. A $P$ value of 0.05 or less was considered significant.

\section{Results}

3.1. Diabetic Wounds Heal Faster in Mice with Low Levels of Flii Gene Expression. To assess the biological function of Flii and determine the effect of Flii gene modulation on diabetic wound healing, three lines of mice were used expressing low $\left(\right.$ Flii $^{+/-}$), normal (Flii ${ }^{+/+}$), and high (Flii ${ }^{\mathrm{Tg} / \mathrm{Tg}}$ ) levels of Flii. Representative digital images of wounds at 7 days subsequent to wounding are shown in Figure 1(A). Overexpression of Flii in diabetic and nondiabetic wounds resulted in delayed wound closure at day 7 following wounding (Figure 1(A)). In contrast, wound area was decreased significantly when Flii levels were reduced (Flii ${ }^{+/-} ; P=0.01$ ) compared to Flii ${ }^{\mathrm{Tg} / \mathrm{Tg}}$ mice at day 7 following wounding (Figure 1(B)(a)). Representative microscopic images of day 7 wounds are shown in Figure 1(A). Histological assessment of these diabetic mouse wounds showed that at day 7 the dermal wound gape was significantly smaller in Flii-deficient mice (Flii ${ }^{+-}$) compared with Flii overexpressing (Flii ${ }^{\mathrm{Tg} / \mathrm{Tg}}$ ) mice (Figure $1(\mathrm{~B})(\mathrm{b}) ; P=$ 0.05).

3.2. Treatment of Diabetic Wounds with Flii Neutralising Antibodies (FnAbs) Improves Wound Healing. Previous studies have shown that intradermal injection of Flii neutralising antibodies reduces the level of Flii in wounds and improves healing [26]. FnAbs were injected intradermally at days 0,1 , and 2 , and representative images of macroscopic (at days 0 and 7) and microscopic (day 7) appearances of diabetic wounds are shown in Figure 2(A). Intradermal administration of FnAb to diabetic wounds resulted in a 1.9fold decrease in average wound area (Figure 2 (B)(a)) and histological wound gape (Figure 2(B)(b)) compared to IgGtreated WT diabetic controls (Figure 2(B) (a) and (b); $P \leq$ 0.05; IgG versus FnAb).

\subsection{Elevating Flii Gene Expression Increases TLR4 in Dia-} betic Mouse Wounds. Immunofluorescence staining of day 7 diabetic wounds in Flii ${ }^{+/-}$, WT, and Flii ${ }^{\mathrm{Tg} / \mathrm{Tg}}$ mice shows increased Flii in Flii ${ }^{\mathrm{Tg} / \mathrm{Tg}}$ diabetic wounds $>\mathrm{WT}>\mathrm{Flii}^{+/-}$ wounds (Figure 3(A)) with significantly less Flii staining being observed in day 7 diabetic $\mathrm{Flii}^{+/-}$and WT wounds $\left(P=0.04\right.$ Flii $^{+/-}$versus $\mathrm{Flii}^{\mathrm{Tg} / \mathrm{Tg}} ; P=0.05$; WT versus $\mathrm{Flii}^{\mathrm{Tg} / \mathrm{Tg}}$; Figure $\left.3(\mathrm{~B})(\mathrm{a})\right)$. The presence of foreign molecules and pathogens was detected by a family of receptors known as toll-like receptors (TLRs), which contribute to prolonged inflammation [27]. To assess if altered levels of Flii affected TLR4 expression, diabetic wounds were stained for TLR4. Increasing Flii leads to a concomitant increase in TLR4 expression (Figure 3(A)(d)-(f)). Flii deficiency caused a significant reduction in TLR4 expression (Figure $3(\mathrm{~B})(\mathrm{b})$ ) 

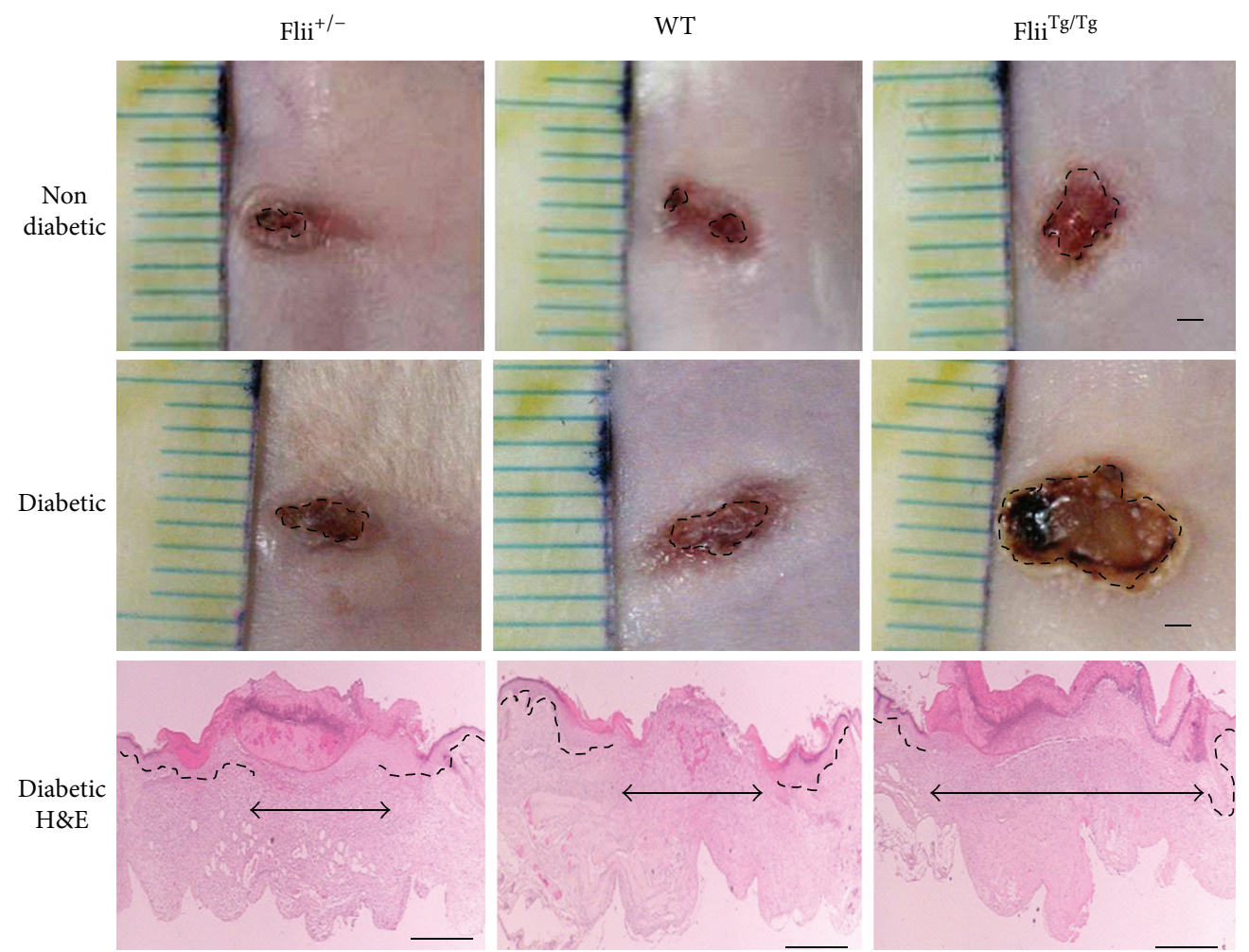

(A)
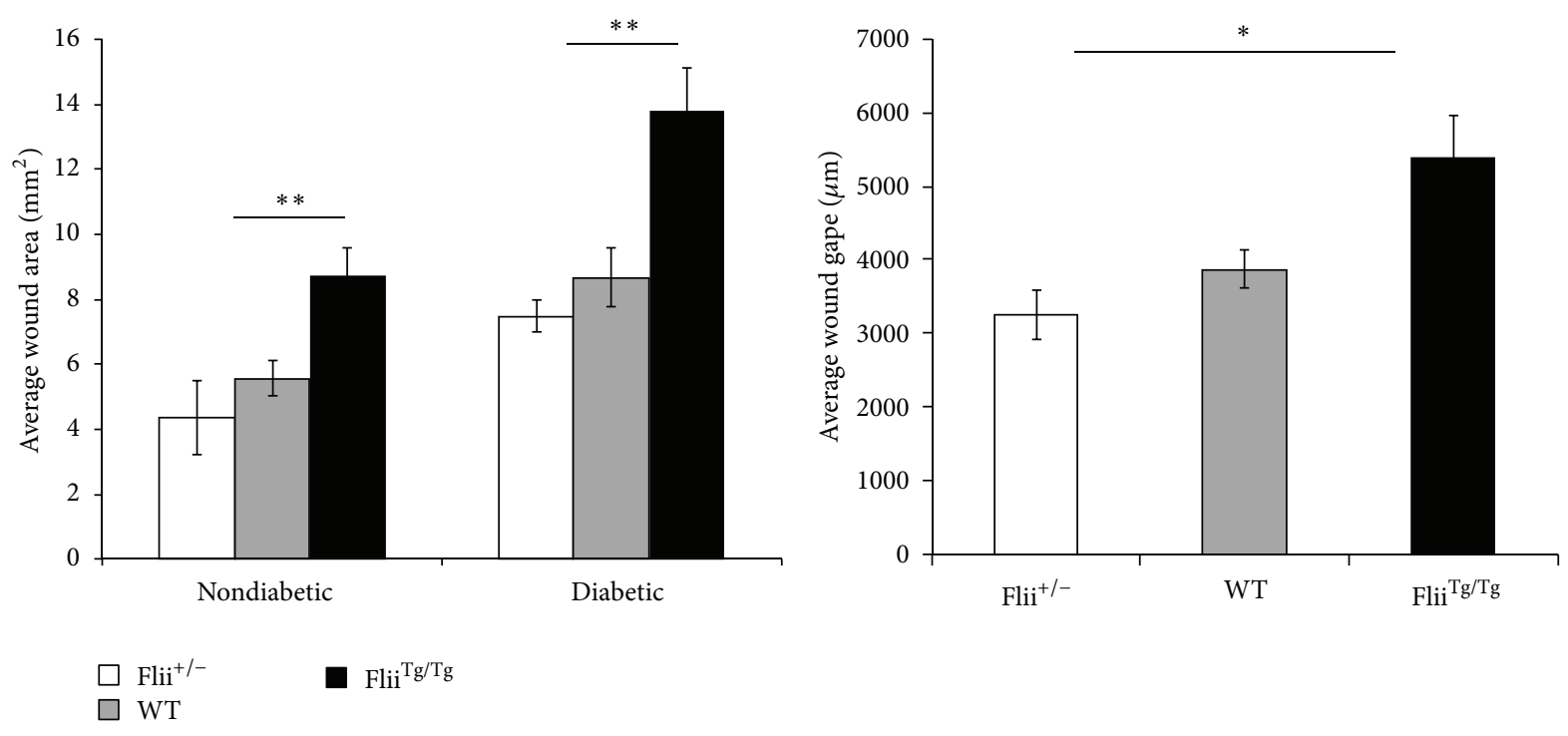

(a)

(B)

(b)

FIGURE 1: Flii delays wound healing in diabetic murine wounds after 7 days.(A) Macroscopic images of day 7 nondiabetic and diabetic mouse wounds in the Flii ${ }^{+/-}$, WT, and Flii ${ }^{\mathrm{Tg} / \mathrm{Tg}}$ mice and representative histological H\&E staining of day 7 diabetic wounds. Scale bars $=1 \mathrm{~mm}$ for macroscopic pictures, and for the histology magnification was $\times 4$ and scale bars $=100 \mu \mathrm{m}$. (B) shows graphical representations of the wound areas of the day 7 (a) wound areas of the three Flii genotype diabetic wounds $(n=6)$ and (b) the microscopic dermal wound gape of the day 7 diabetic wounds where ${ }^{* *} P \leq 0.01$ and ${ }^{*} P \leq 0.05(n=6)$.

at day 7 following wounding which was significantly lower than WT and Flii ${ }^{\mathrm{Tg} / \mathrm{Tg}}$ wounds $\left(P \leq 0.01 \mathrm{WT}\right.$ versus Flii $^{+/-}$; $P \leq 0.001$ WT versus Flii $\left.{ }^{\mathrm{Tg} / \mathrm{Tg}}\right)$. Given that the inflammatory response in diabetic wounds was associated with increased
TLR4 expression, we next proceeded to test if the downstream molecule NF- $\kappa$ B production was also affected. Figure $3(\mathrm{~A})$ shows that NF- $\kappa$ B expression was also increased in Flii overexpressing wounds (5-fold higher than WT diabetic wounds) 
D0
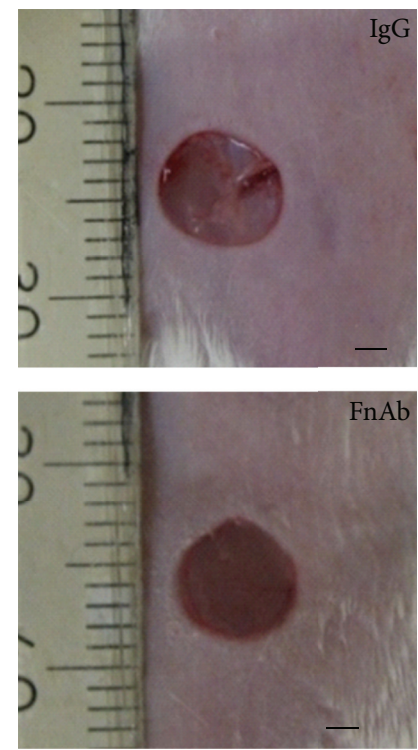

Wound area

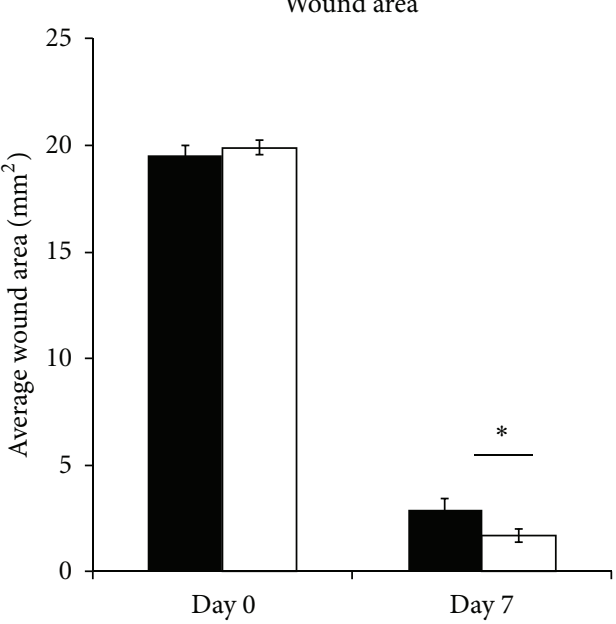

$\square \mathrm{IgG}$

- FnAb
D7
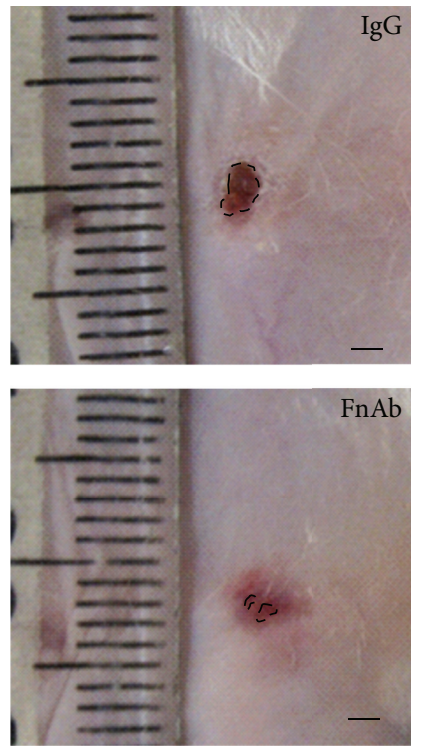

(A)

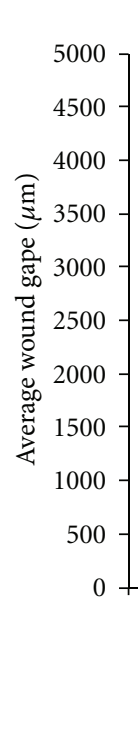

(B)
D7
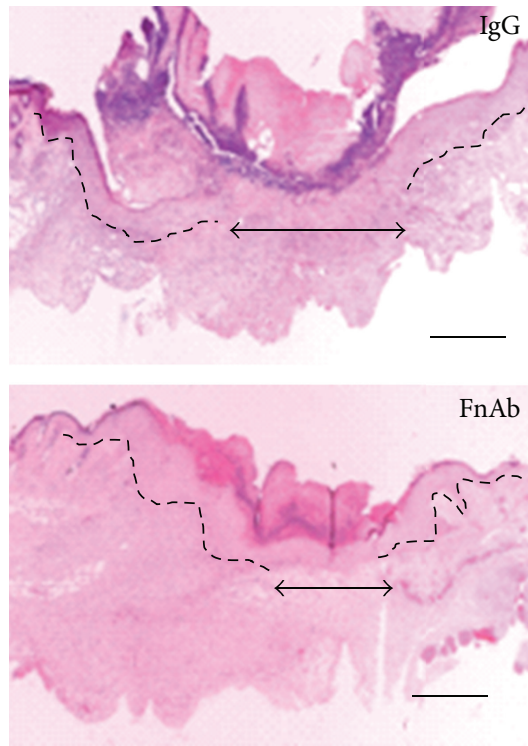

Wound gape

(a)

(b)

Figure 2: Healing can be improved by the application of Flii neutralizing antibodies. (A) shows macroscopic images of day 0 and day 7 excisional WT diabetic wounds treated with IgG isotype control $(n=10)$ and Flii neutralizing antibody $($ FnAb $)(n=10)$. Representative pictures of H\&E staining of day 7 diabetic wounds treated with IgG and FnAb. (B) Graphical representation of the (a) wound areas and (b) histological wound gapes of the days 0 and 7 IgG control-treated and FnAb-treated WT diabetic wounds where ${ }^{*} P \leq 0.05(n=10)$.

(Figure $3(\mathrm{~B})(\mathrm{c}) ; P \leq 0.001 \mathrm{Flii}^{\mathrm{Tg} / \mathrm{Tg}}$ versus WT). A reduction in NF- $\kappa$ B was observed between $\mathrm{Flii}^{+/-}$wounds and WT, but this was not statistically significant (Figure 3(B)(c)).

3.4. Flii Neutralising Antibodies Reduce TLR4 Expression in Diabetic Wounds. Given that intradermal application of $\mathrm{FnAb}$ resulted in improved healing of diabetic wounds
(Figure 2(A)), we proceeded to test whether this was in part due to modulated levels of TLR4-mediated inflammation. TLR4 and NF- $\kappa$ B expression was quantified, and representative images are shown in Figure 4(A). Treatment with FnAbs resulted in a significant decrease in TLR4 expression (Figure $4(\mathrm{~B}) ; P \leq 0.05 \mathrm{IgG}$ versus $\mathrm{FnAb}$ ) whereas NF$\kappa \mathrm{B}$ expression in day 7 FnAb-treated wounds remained unchanged compared to IgG controls (Figure 4(B)(b)). 


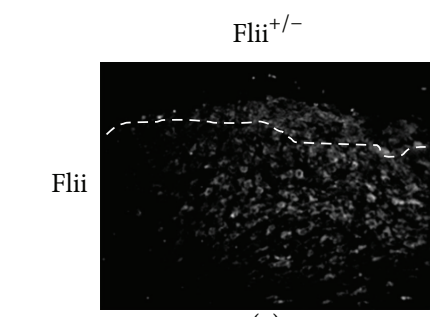

(a)

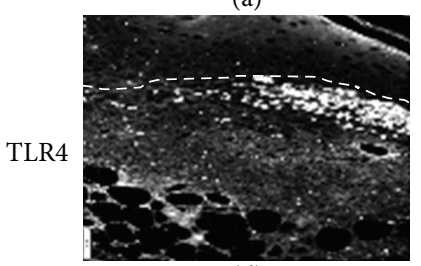

(d)

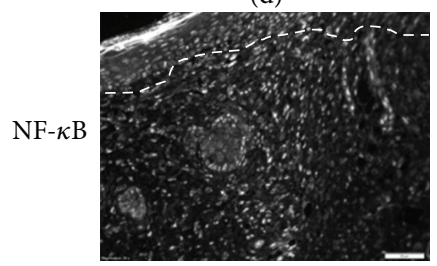

(g)

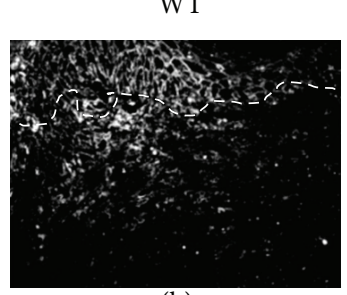

(b)

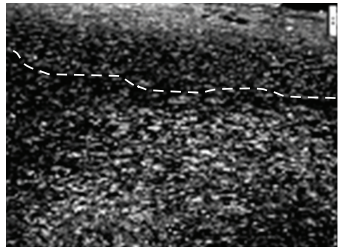

(e)

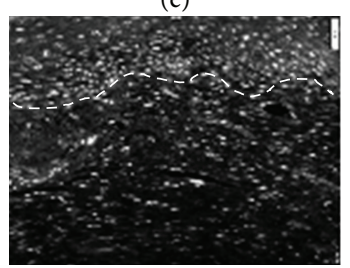

(h)

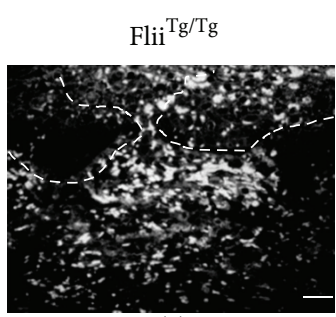

(c)

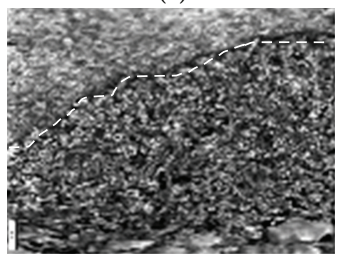

(f)

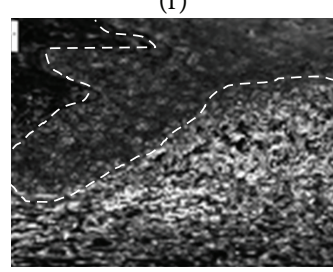

(i)

(A)

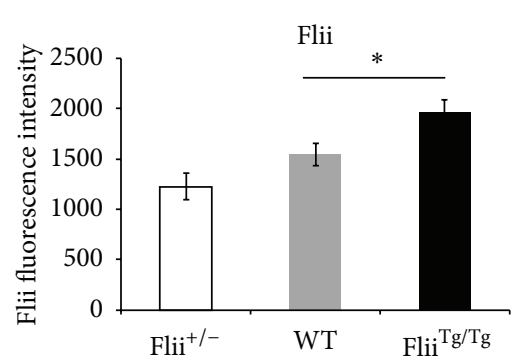

(a)

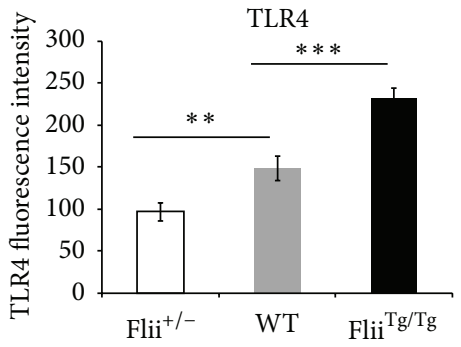

(b)

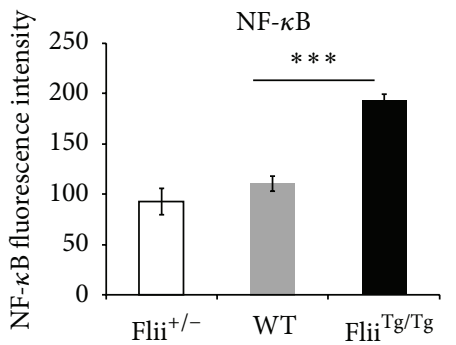

(c)

(B)

FIGURE 3: Concomitant increase in TLR4 and NF- $\kappa$ B staining occurs with increasing levels of Flii. (A) Representative images for Flii (a)-(c), TLR4 (d)-(f), and NF- $\kappa$ B (g)-(i) immunostaining of the three Flii genotypes (Flii ${ }^{+/-}$, WT, and Flii ${ }^{\mathrm{Tg} / \mathrm{Tg}}$ ). (B) Graphical representation of (a) Flii, (b) TLR4, and (c) NF- $\kappa$ B in Flii ${ }^{+/-}$, WT, and Flii ${ }^{\mathrm{Tg} / \mathrm{Tg}}$ day 7 diabetic wounds. ${ }^{*} P \leq 0.05{ }^{* *} P \leq 0.01{ }^{* * *} P \leq 0.001(n=6)$ and scale bar $=$ $100 \mu \mathrm{m}$.

\section{Discussion}

Flii has been identified as a protein that can inhibit the rate of healing by reducing the migration of keratinocytes and fibroblasts and limiting the degree of wound contraction $[18,22,28]$. Flii deficiency is associated with improved reepithelialisation in acute wounds [18] while Flii overexpressing mice have impaired wound healing with delayed reepithelialisation. Here we have investigated if Flii is involved in the impaired healing associated with diabetic wounds. Wound healing was impaired as Flii levels increased, and this impairment was exacerbated when diabetes was induced. Flii is upregulated during the wound repair process $[18,25]$ and is constitutively secreted by two of the major cell types found in wounds: fibroblasts and macrophages in response to wounding both in vitro [29] and in vivo [22, 30]. Recent studies show that Flii is also secreted through a nonclassical late endocytic/lysosomal pathway of secretion by fibroblasts and macrophages [29]. Addition of Flii monoclonal neutralising antibodies as a means of reducing the Flii protein in the wound environment was able to counteract the negative effect of Flii on wound healing [18], and treatment of murine diabetic wounds with neutralising antibodies to Flii led to an improvement in healing suggesting that high levels of Flii in diabetic wounds contribute to wound chronicity.

Inflammation is an essential component of the normal wound healing process; however excessive inflammation is detrimental to this process [31]. Disproportionate inflammation is one of the major contributing factors to the formation of diabetic ulcers as these chronic wounds often have an unregulated and excessive inflammatory reaction [31, 32]. The innate immune system detects foreign particles such as 

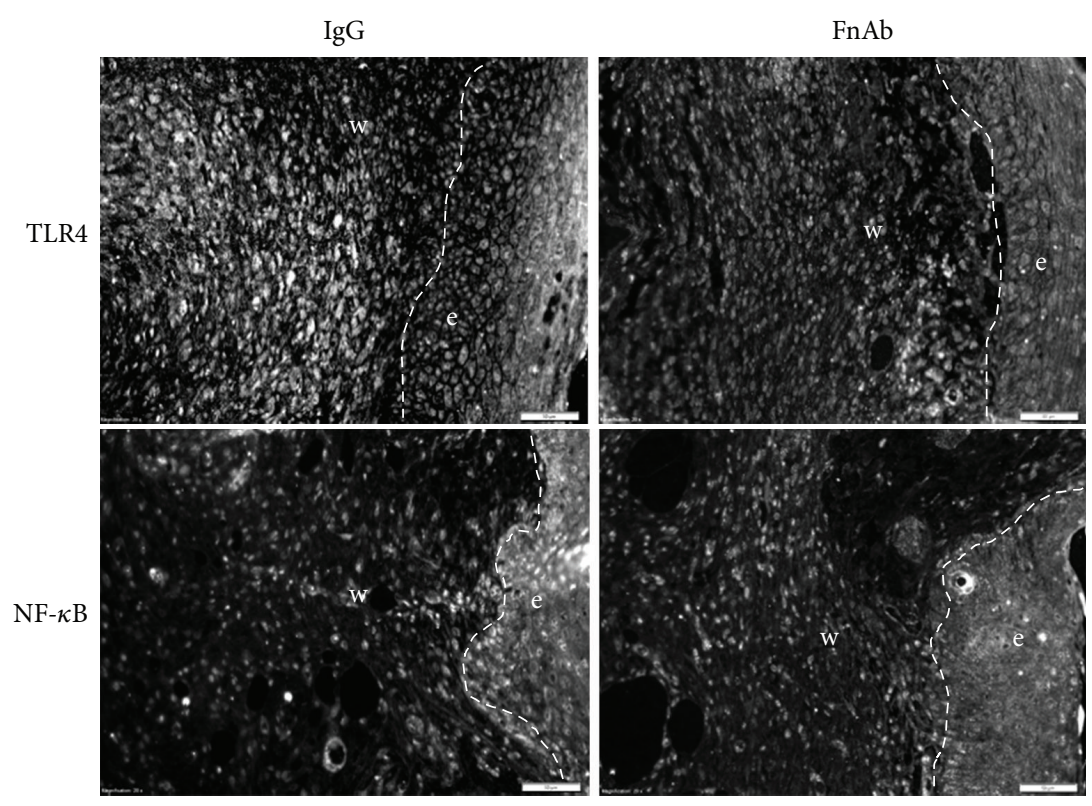

(A)

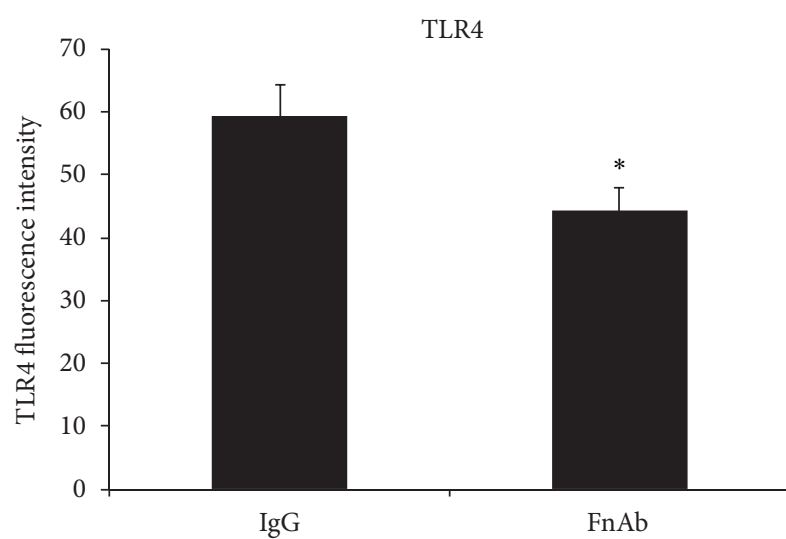

(a)

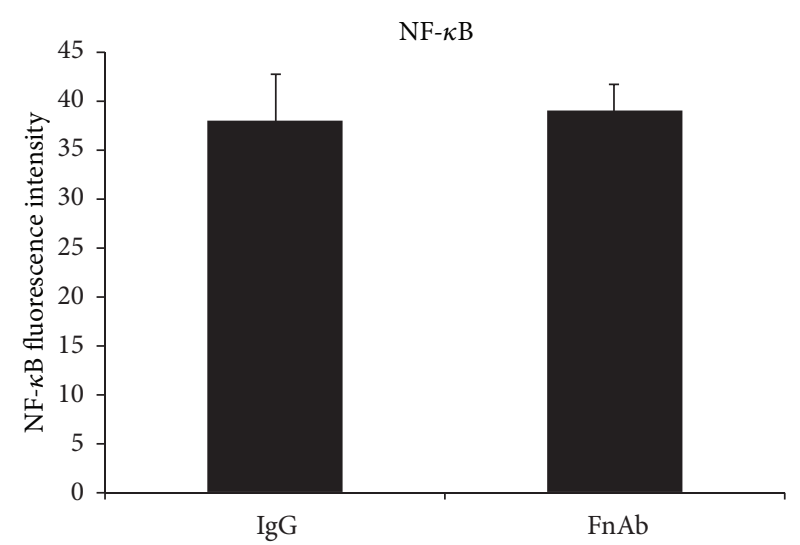

(b)

(B)

FIgURE 4: Modulation of Flii by exogenous application of Flii neutralising antibodies (FnAbs) reduces TLR4 expression in diabetic wounds. (A) Representative images of TLR 4 and NF- $\kappa$ B staining of day 7 diabetic wounds treated with IgG and FnAb. (B) Graphical representation of (a) TLR4 and (b) NF- $\kappa$ B staining of day 7 diabetic wounds treated with IgG and FnAb. ${ }^{*} P \leq 0.05$.

bacteria, fungi, and viruses via pathogen-associated molecular pattern (PAMP) molecules leading to the activation of the inflammatory response via toll-like receptors (TLRs) which recognise PAMPs $[33,34]$. TLRs have also been linked to diabetes with studies showing that TLR-immune activation can result in activation of proinflammatory pathways leading to autoimmunity which may cause the onset of diabetes [27]. Previous reports suggested that stimulation of TLR4 leads to activation of the downstream transcriptional regulator NF- $\kappa \mathrm{B}$ resulting in cytokine secretion [21], and both TLR4 expression and pro-inflammatory cytokine NF$\kappa \mathrm{B}$ were elevated in the murine diabetic wounds which would further contribute to inflammation and chronicity. In Fliideficient diabetic mouse wounds, decreased TLR4 and NF$\kappa \mathrm{B}$ were observed and when Flii neutralising antibodies were administered to diabetic wounds, a decrease in TLR4 expression was seen suggesting a dampening of the inflammatory response when Flii levels were reduced. Interestingly, the effect of FnAb was specific to TLR4 and did not appear to affect the production of the proinflammatory cytokine NF$\kappa \mathrm{B}$ suggesting that alternative pathways may still be active in these wounds.

In vitro studies have previously shown that the LRR region of Flii shares $29 \%$ sequence identity and $42 \%$ similarity to TLR4 [20] suggesting that Flii may influence TLR signalling. However, while in vitro studies showed that through its interaction with MyD88, Flii was able to negatively regulate the TLR4-MyD88-mediated activation of NF- $\kappa \mathrm{B}$ and the subsequent cytokine secretion in macrophages [20,21], our studies show that in vivo, in mouse diabetic wounds the 
increased levels of Flii appear to correlate with an increase in the expression of TLR4 and its signalling protein NF- $\kappa \mathrm{B}$. This is clearly in opposition to the findings in these cell-based studies but may be accounted for by the differing in vivo and in vitro environments. The time points investigated also differ with the in vitro studies looking at responses from 3 to 6 hours whereas this wounding study investigated time points which were measured in days rather than hours. It is, however, not inconceivable that Flii may have a dual role in wounding and in the inflammatory response which depends on the time point investigated.

In conclusion, inflammation is an integral component of the normal wound healing process and occurs even in the absence of infection; however excessive and prolonged inflammation impairs healing. Flii is a multifunctional protein and is currently emerging as a regulator of inflammation; however, whether it is pro-or anti-inflammatory is still to be determined. Our in vivo studies show that reducing the expression of Flii in diabetic murine wounds improves healing and reduces the proinflammatory response. Being able to manipulate the level of inflammation in a wound would greatly improve the wound healing outcomes of patients with diabetes, and it remains to be elucidated whether neutralisation of Flii in human diabetic wounds could help improve healing of these chronic nonhealing ulcers.

\section{Conflict of Interests}

The authors know of no potential conflict of interests.

\section{Acknowledgment}

A. J. Cowin is supported by NHMRC Senior Research Fellowship (no. 1002009).

\section{References}

[1] A. J. Boulton, L. Vileikyte, G. Ragnarson-Tennvall, and J. Apelqvist, "The global burden of diabetic foot disease," The Lancet, vol. 366, no. 9498, pp. 1719-1724, 2005.

[2] D. G. Armstrong, J. Wrobel, and J. M. Robbins, "Guest editorial: are diabetes-related wounds and amputations worse than cancer?" International Wound Journal, vol. 4, no. 4, pp. 286-287, 2007.

[3] M. Goshima, K. I. Kariya, Y. Yamawaki-Kataoka et al., "Characterization of a novel ras-binding protein Ce-FLI-1 comprising leucine-rich repeats and gelsolin-like domains," Biochemical and Biophysical Research Communications, vol. 257, no. 1, pp. 111-116, 1999.

[4] K. S. K. Fong and H. G. de Couet, "Novel proteins interacting with the leucine-rich repeat domain of human flightless-I identified by the yeast two-hybrid system," Genomics, vol. 58, no. 2, pp. 146-157, 1999.

[5] Z. Kopecki and A. J. Cowin, "Flightless I: an actin-remodelling protein and an important negative regulator of wound repair," International Journal of Biochemistry and Cell Biology, vol. 40, no. 8, pp. 1415-1419, 2008.

[6] D. A. Davy, E. E. Ball, K. I. Matthaei, H. D. Campbell, and M. F. Crouch, "The flightless I protein localizes to actin-based structures during embryonic development," Immunology and Cell Biology, vol. 78, no. 4, pp. 423-429, 2000.

[7] D. A. Davy, H. D. Campbell, S. Fountain, D. De Jong, and M. F. Crouch, "The flightless I protein colocalizes with actin- and microtubule-based structures in motile Swiss 3T3 fibroblasts: evidence for the involvement of PI 3-kinase and Ras-related small GTPases," Journal of Cell Science, vol. 114, no. 3, pp. 549$562,2001$.

[8] K. L. Straub, M. C. Stella, and M. Leptin, “The gelsolin-related flightless I protein is required for actin distribution during cellularisation in Drosophila," Journal of Cell Science, vol. 109, no. 1, pp. 263-270, 1996.

[9] S. A. Wilson, E. C. Brown, A. J. Kingsman, and S. M. Kingsman, "TRIP: a novel double stranded RNA binding protein which interacts with the leucine rich repeat of Flightless I," Nucleic Acids Research, vol. 26, no. 15, pp. 3460-3467, 1998.

[10] S. K. Archer, C. A. Behm, C. Claudianos, and H. D. Campbell, "The flightless I protein and the gelsolin family in nuclear hormone receptor-mediated signalling," Biochemical Society Transactions, vol. 32, no. 6, pp. 940-942, 2004.

[11] Y. H. Lee, H. D. Campbell, and M. R. Stallcup, "Developmentally essential protein flightless $\mathrm{I}$ is a nuclear receptor coactivator with actin binding activity," Molecular and Cellular Biology, vol. 24, no. 5, pp. 2103-2117, 2004.

[12] Y. H. Lee and M. R. Stallcup, "Interplay of Fli-I and FLAP1 for regulation of $\beta$-catenin dependent transcription," Nucleic Acids Research, vol. 34, no. 18, pp. 5052-5059, 2006.

[13] T. Wang, T. H. Chuang, T. Ronni et al., "Flightless I homolog negatively modulates the TLR pathway," Journal of Immunology, vol. 176, no. 3, pp. 1355-1362, 2006.

[14] P. G. Dai, S. Y. Jeong, Y. Yu et al., "Modulation of TLR signaling by multiple MyD88-interacting partners including leucine-rich repeat Fli-I-interacting proteins," Journal of Immunology, vol. 182, no. 6, pp. 3450-3460, 2009.

[15] H. Deng, D. Xia, B. Fang, and H. Zhang, "The flightless I homolog, fli-1, regulates anterior/posterior polarity, asymmetric cell division and ovulation during Caenorhabditis elegans development," Genetics, vol. 177, no. 2, pp. 847-860, 2007.

[16] M. E. Seward, C. A. Easley, J. J. McLeod, A. L. Myers, and R. M. Tombes, "Flightless-I, a gelsolin family member and transcriptional regulator, preferentially binds directly to activated cytosolic CaMK-II," FEBS Letters, vol. 582, no. 17, pp. 24892495, 2008.

[17] J. Y. Li, H. L. Yin, and J. Y. Yuan, “Flightless-I regulates proinflammatory caspases by selectively modulating intracellular localization and caspase activity," Journal of Cell Biology, vol. 181, no. 2, pp. 321-333, 2008.

[18] A. J. Cowin, D. H. Adams, X. L. Strudwick et al., "Flightless I deficiency enhances wound repair by increasing cell migration and proliferation," Journal of Pathology, vol. 211, no. 5, pp. 572581, 2007.

[19] D. A. Davy, H. D. Campbell, S. Fountain, D. De Jong, and M. F. Crouch, "The flightless I protein colocalizes with actin- and microtubule-based structures in motile Swiss 3T3 fibroblasts: evidence for the involvement of PI 3-kinase and Ras-related small GTPases," Journal of Cell Science, vol. 114, part 3, pp. 549562, 2001.

[20] T. Wang, T. H. Chuang, T. Ronni et al., "Flightless I homolog negatively modulates the TLR pathway," Journal of Immunology, vol. 176, no. 3, pp. 1355-1362, 2006.

[21] P. Dai, S. Y. Jeong, Y. Yu et al., "Modulation of TLR signaling by multiple MyD88-interacting partners including leucine-rich 
repeat Fli-I-interacting proteins," Journal of Immunology, vol. 182, no. 6, pp. 3450-3460, 2009.

[22] J. E. Jackson, Z. Kopecki, D. H. Adams, and A. J. Cowin, "Flii neutralizing antibodies improve wound healing in porcine preclinical studies," Wound Repair Regen, vol. 20, no. 4, pp. 523536, 2012.

[23] H. D. Campbell, S. Fountain, I. S. McLennan et al., "Fliih, a gelsolin-related cytoskeletal regulator essential for early mammalian embryonic development," Molecular and Cellular Biology, vol. 22, no. 10, pp. 3518-3526, 2002.

[24] M. S. Johnson, J. M. Ryals, and D. E. Wright, "Early loss of peptidergic intraepidermal nerve fibers in an STZ-induced mouse model of insensate diabetic neuropathy," Pain, vol. 140, no. 1, pp. 35-47, 2008.

[25] D. H. Adams, N. Ruzehaji, X. L. Strudwick et al., "Attenuation of Flightless I, an actin-remodelling protein, improves burn injury repair via modulation of transforming growth factor (TGF)betal and TGF-beta3," The British Journal of Dermatology, vol. 161, no. 2, pp. 326-336, 2009.

[26] D. H. Adams, N. Ruzehaji, X. L. Strudwick et al., "Attenuation of Flightless I, an actin-remodelling protein, improves burn injury repair via modulation of transforming growth factor (TGF)betal and TGF-beta3," The British Journal of Dermatology, vol. 161, no. 2, pp. 326-336, 2009.

[27] A. Marshak-Rothstein, "Toll-like receptors in systemic autoimmune disease," Nature Reviews Immunology, vol. 6, no. 11, pp. 823-835, 2006.

[28] D. H. Adams, X. L. Strudwick, Z. Kopecki et al., "Gender specific effects on the actin-remodelling protein Flightless I and TGF- $\beta 1$ contribute to impaired wound healing in aged skin," International Journal of Biochemistry and Cell Biology, vol. 40, no. 8, pp. 1555-1569, 2008.

[29] N. Lei, L. Franken, N. Ruzehaji, C. Offenhäuser, A. J. Cowin, and R. Z. Murray, "Flightless, secreted through a late endosome/lysosome pathway, binds LPS and dampens cytokine secretion," Journal of Cell Science, vol. 125, part 18, pp. 42884296, 2012.

[30] N. Ruzehaji, R. Grose, D. Krumbiegel, H. Zola, R. Fitridge, and A. J. Cowin, "Cytoskeletal protein Flightless (Flii) is elevated in chronic and acute human wounds and wound fluid: neutralizing its activity in chronic but not acute wound fluid improves cellular proliferation," European Journal of Dermatology, vol. 22, no. 6, pp. 740-750, 2012.

[31] B. S. Pukstad, L. Ryan, T. H. Flo et al., "Non-healing is associated with persistent stimulation of the innate immune response in chronic venous leg ulcers," Journal of Dermatological Science, vol. 59, no. 2, pp. 115-122, 2010.

[32] N. B. Menke, K. R. Ward, T. M. Witten, D. G. Bonchev, and R. F. Diegelmann, "Impaired wound healing," Clinics in Dermatology, vol. 25, no. 1, pp. 19-25, 2007.

[33] Q. Lin, M. Li, D. Fang, J. Fang, and S. B. Su, “The essential roles of Toll-like receptor signaling pathways in sterile inflammatory diseases," International Immunopharmacology, vol. 11, no. 10, pp. 1422-1432, 2011.

[34] L. A. J. O'Neill and A. G. Bowie, "The family of five: TIRdomain-containing adaptors in Toll-like receptor signalling," Nature Reviews Immunology, vol. 7, no. 5, pp. 353-364, 2007. 


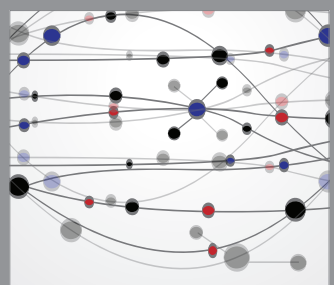

The Scientific World Journal
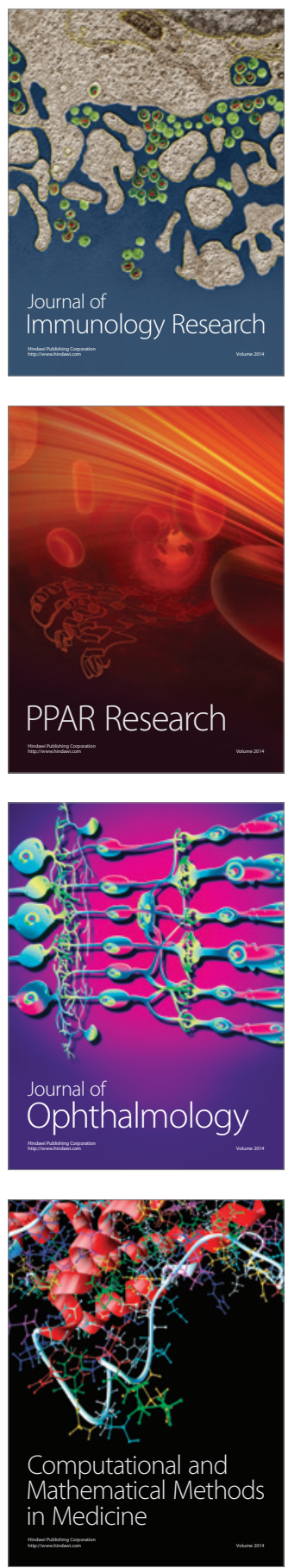

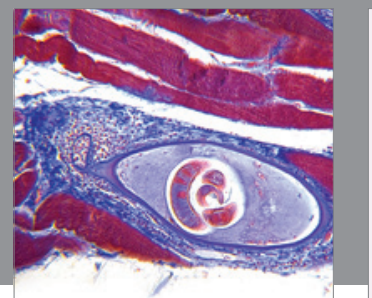

Gastroenterology

Research and Practice
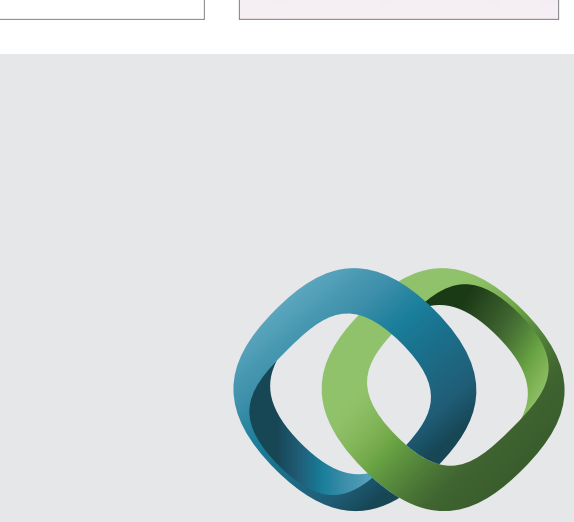

\section{Hindawi}

Submit your manuscripts at

http://www.hindawi.com
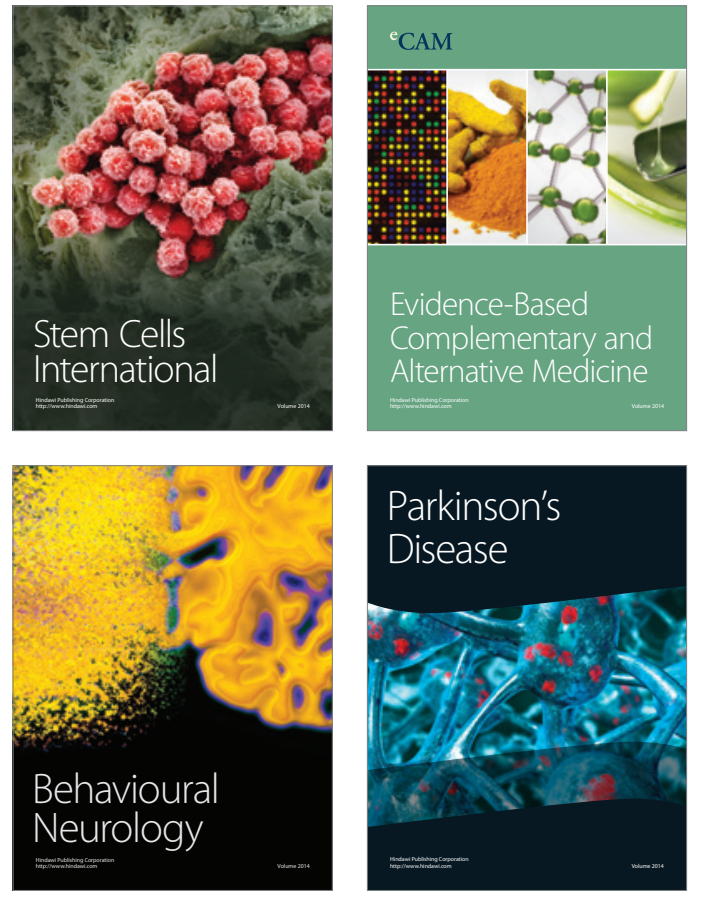
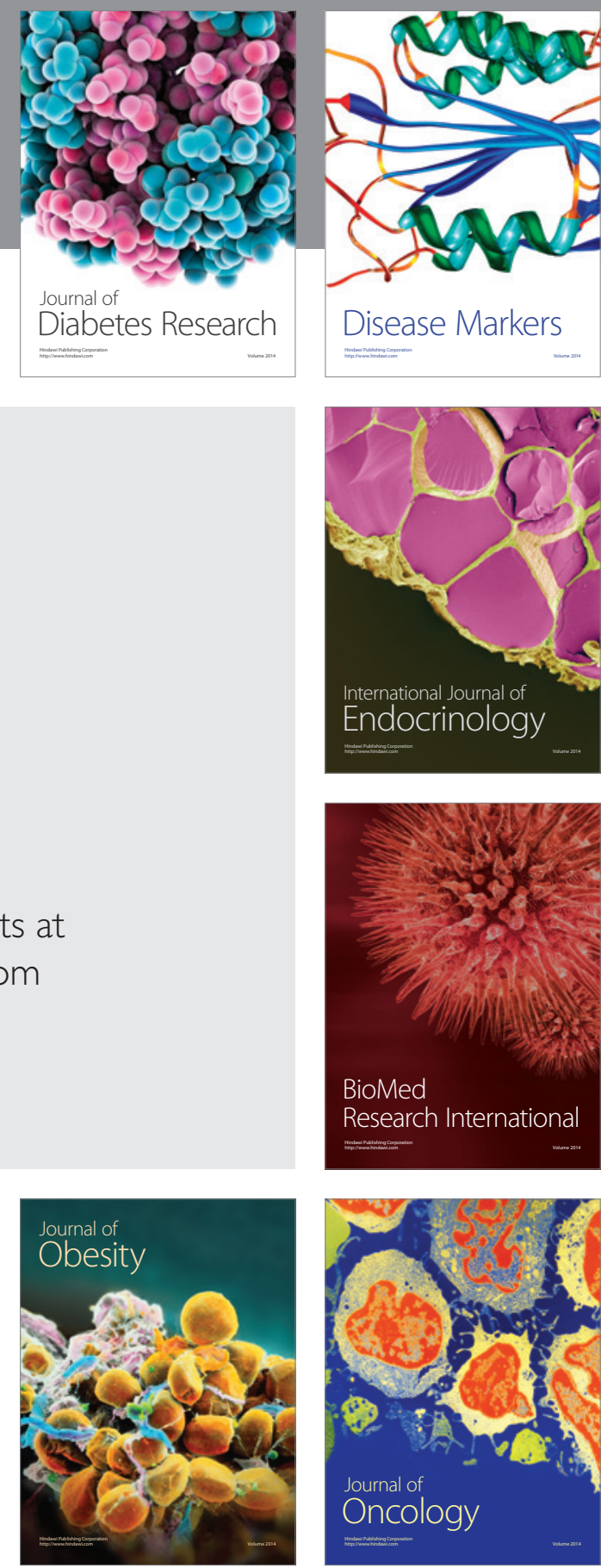

Disease Markers
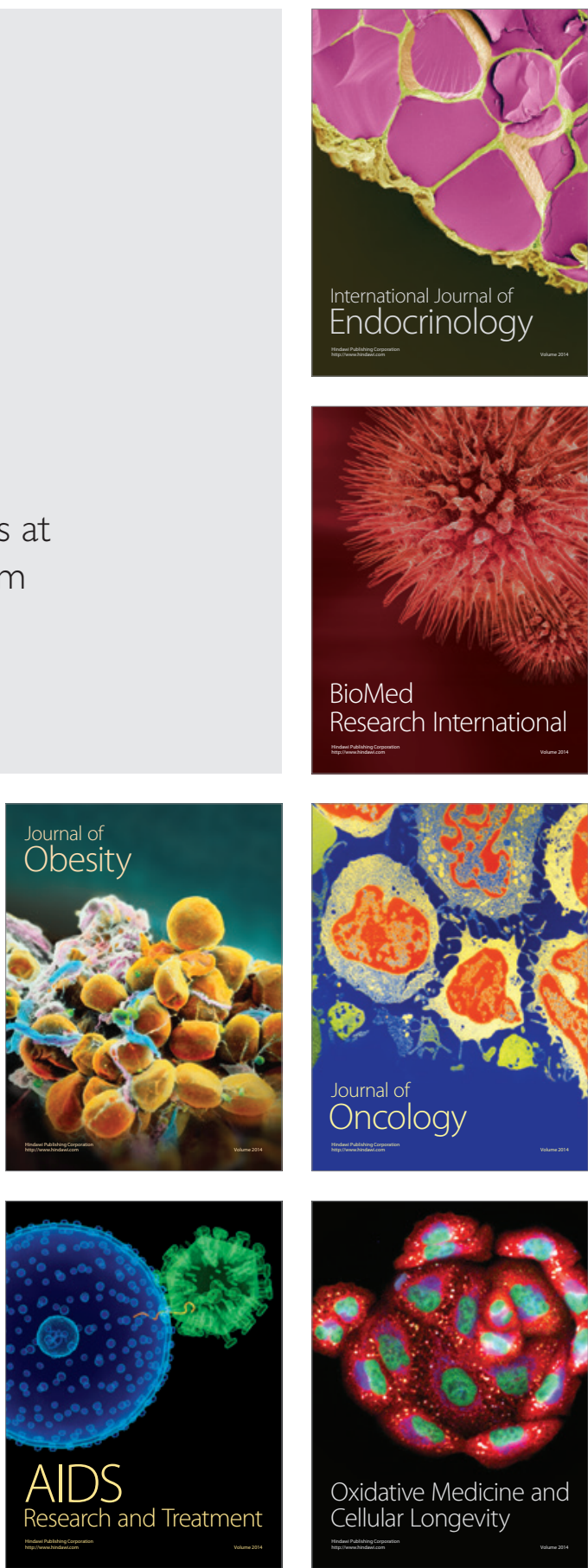